\title{
A responsabilização do Programa de Segurança do Paciente: um novo olhar pela equipe de Enfermagem
}

\author{
Accountability of the Patient Safety Program: a new look by the Nursing team
}

\author{
Responsabilidad del programa de seguridad del paciente: una nueva mirada del equipo de enferme- \\ ría
}

\section{Rafaela Barboza Arantes ${ }^{*}$, Daniele Viana Alves ${ }^{2}$, Alana Amancio Consentino ${ }^{3}$, Denize Duarte Celento $^{4}$}

Como citar esse artigo. Arantes, RB; Alves, DV; Consentino, AA; Celento, DC. A responsabilização do Programa de Segurança do Paciente: um novo olhar pela equipe de Enfermagem. Revista Pró-UniverSUS. 2021 Jan./Jun.; 12 (1): 09-13.

\section{Resumo}

A Segurança do Paciente hoje é considerada um dos atributos de qualidade e visa prover um cuidado seguro e efetivo aos usuários dos serviços de saúde, prevê a cultura de segurança do paciente que pode ser entendida como produto de valores, atitudes, percepções e competências coletivas e individuais, que substituem a culpa e a punição pela oportunidade de aprender, algo que vem tentando ser implementado nas instituições. Desta forma, este presente estudo tem como objetivo reavaliar a cultura de responsabilização em ambiente hospitalar visando traçar uma correlação entre "erros" e "riscos". Trata-se de uma pesquisa de natureza qualitativa de caráter exploratório realizada com a participação de 08 enfermeiros em dois momentos distintos: inicialmente em um Hospital Geral de Ensino do Interior do Estado do RJ e posteriormente por meio de uma ferramenta de videoconferência. A Coleta de dados foi realizada pela técnica de entrevista em grupo focal. Após a análise da fala dos participantes pode-se concluir que a segurança do paciente ainda é um campo pouco conhecido pelos profissionais desta referida instituição e que mesmo que haja um clima favorável a implementação da cultura de segurança do paciente, os profissionais ainda manifestam o medo de errar, pôde-se perceber também que o Núcleo de Segurança do Paciente ainda não é visto como uma instância de gestão, portanto sugerimos mediante a explanação dos enfermeiros que o NSP busque a revisão em suas atribuições e atividade de gestão, por fim ressaltamos que o estudo em segurança do paciente não se esgota nesse momento.

Palavras-chave: Segurança do Paciente; Cultura de Segurança do Paciente; Qualidade da Assistência; Cultura de Responsabilização.

\begin{abstract}
Patient Safety today is considered one of the attributes of quality and aims to provide safe and effective care to users of health services, provides for a culture of patient safety that can be understood as a product of collective values, attitudes, perceptions and competences and that substitute guilt and punishment for the opportunity to learn, something that has been trying to be implemented in institutions. Thus, this study aims to reevaluate the culture of accountability in a hospital environment in order to trace a correlation between "errors" and "risks". This is a qualitative research of an exploratory nature carried out with the participation of 08 nurses in two different moments: initially in a General Hospital for Teaching of the Interior of the State of RJ and later through a videoconference tool. Data collection was performed using the focus group interview technique. After analyzing the speech of the participants, it can be concluded that patient safety is still a little-known field by the professionals of this institution and that even if there is a favorable climate for the implementation of the patient safety culture, professionals still express fear to make mistakes, it was also possible to notice that the Patient Safety Center is not yet seen as a management instance, therefore we suggest, through the nurses' explanation, that the NSP look for a review in its attributions and management activity, finally we emphasize that the study on patient safety does not end at that moment.
\end{abstract}

Keywords: Patient Safety; Patient Safety Culture; Quality of Assistance; Culture of Accountability.

${ }^{1}$ Acadêmica do Curso de Enfermagem, Universidade de Vassouras. RJ, Brasil. E-mail: rafaela-barboza@hotmail.comORCIDhttps://orcid.org/0000-0002-1627-6138

${ }^{2}$ Acadêmica do Curso de Enfermagem, Universidade de Vassouras. RJ, Brasil. E-mail: daniviana.alves@hotmail.com ORCID:https://orcid.org/0000-0002-9936-3455

${ }^{3}$ Acadêmica do Curso de Enfermagem, Universidade de Vassouras. RJ, Brasil. E-mail:alana.consentino@hotmail.com ORCID: ORCID: https://orcid.org/0000-0003-1064-8671

${ }^{4}$ Administradora de Empresas. Mestre em Ciências do Cuidado em Saúde. Docente do Curso de Enfermagem da Universidade de Vassouras. RJ, Brasil. E-mail:denize.celento@,

hotmail.com ORCID: https://orcid.org/0000-0002-1829-759X 


\section{Resumen}

La Seguridad del Paciente hoy es considerada uno de los atributos de la calidad y tiene como objetivo brindar una atención segura y eficaz a los usuarios de los servicios de salud, prevé una cultura de seguridad del paciente que puede entenderse como producto de valores, actitudes, percepciones y competencias colectivas que sustituyen la oportunidad de aprender por la culpa y el castigo, algo que se viene intentando implementar en las instituciones. Por lo tanto, este estudio tiene como objetivo reevaluar la cultura de la responsabilidad en un entorno hospitalario para trazar una correlación entre "errores" y "riesgos". Se trata de una investigación cualitativa de carácter exploratorio que se llevó a cabo con la participación de 08 enfermeros en dos momentos diferentes: inicialmente en un Hospital General de la Docencia del Interior del Estado de RJ y posteriormente mediante una herramienta de videoconferencia. La recolección de datos se realizó mediante la técnica de entrevista de grupo focal. Luego de analizar el discurso de los participantes, se puede concluir que la seguridad del paciente es aún un campo poco conocido por los profesionales de esta institución y que aun si existe un clima favorable para la implementación de la cultura de seguridad del paciente, los profesionales aún expresan temor. Para cometer errores, también se pudo notar que el Centro de Seguridad del Paciente aún no es visto como una instancia gerencial, por lo que sugerimos, a través de la explicación de las enfermeras, que el NSP busque una revisión de sus atribuciones y actividad gerencial, finalmente enfatizamos que el estudio sobre seguridad del paciente no termina en ese momento.

Palabras clave: Seguridad del Paciente; Cultura de Seguridad del Paciente; Calidad de Atención; Cultura de Responsabilidad.

\section{Introdução}

No início deste século, o Instituto de Medicina (IOM) dos Estados Unidos da América (EUA) passou a incorporar "segurança do paciente" como um dos seis atributos da qualidade, com a efetividade, a centralidade no paciente, a oportunidade do cuidado, a eficiência e a equidade. ${ }^{1}$

A Organização Mundial da Saúde (OMS), em 2004, demonstrando preocupação com a situação, criou a World Alliance for Patient Safety. Os objetivos desse programa, (que passou a chamar-se Patient Safety Program) eram, entre outros, organizar os conceitos e as definições sobre segurança do paciente e propor medidas para reduzir os riscos e mitigar os eventos adversos. ${ }^{2,3,4}$

No Brasil em 2013, foi instituído o Programa Nacional de Segurança do Paciente - PNSP, conforme previsto em Portaria do Ministério da Saúde - MS. O PNSP prevê a criação de "núcleos", que são instâncias que devem ser criadas nos serviços de saúde para promover e apoiar a implementação de iniciativas voltadas à segurança do paciente. ${ }^{5}$

Os Núcleos de Segurança do Paciente - NSP hospitalares deverão estar vinculados organicamente a Direção e ter uma agenda permanente e periódica, além de participação nas reuniões das demais instâncias existentes que gerenciam aspectos da qualidade.

Gerir é o ato de governar pessoas e instituições. Portanto, refere-se à capacidade de direcionar os colaboradores à missão organizacional. Para os serviços de saúde é proposta uma atenção gerenciada (managedcare), que visa diminuir os custos e aumentar a eficácia do trabalho.

A Organização Pan-Americana de Saúde, incentiva a produção de conhecimento na área. A entidade exerce papel fundamental na melhoria dos serviços de saúde, pela transferência de tecnologias produzidas pelos países membros e difusão de informações. ${ }^{6}$
A avaliação tecnológica em saúde, aponta as consequências, a curto e longo prazo, da adoção de determinado procedimento. Isso engloba aspectos econômicos e administrativos e otimiza, ao mesmo tempo, recursos financeiros e humanos. A atenção ao paciente merece destaque, visto dentro desse contexto, com aplicabilidade de protocolos e normas de humanização.

A complexidade dos sistemas e serviços de saúde é um fato inegável. Seja pelo trabalho em si ou pelas exigências de uma boa gestão, o profissional tem a responsabilidade de oferecer bons serviços e alcançar resultados de qualidade.

Os profissionais e gestores de saúde estão se dando conta de que houve uma mudança na forma de prestação de cuidados, com o avanço dos conhecimentos científicos e tecnológicos. O cuidado à saúde, que antes era simples, menos efetivo e relativamente seguro, passou a ser mais complexo, mais efetivo, porém potencialmente perigoso.

$\mathrm{O}$ interesse em desenvolver esse estudo emergiu do cenário de vivência, por mais de 25 anos de uma das pesquisadoras. Esse desafio se apresenta no dia a dia das instituições de saúde e na execução das práticas dos profissionais de saúde. Portanto o presente trabalho objetivou reavaliar a cultura de responsabilização em um hospital geral de ensino do interior do estado do RJ; visando a correlação entre "erros" e "riscos".

\section{Metodologia}

Trata-se de uma Pesquisa qualitativa de caráter descritivo exploratório de campo, oriundo de um projeto de pesquisa intitulado "O novo olhar para as responsabilizações do programa de segurança do paciente", aprovado pelo Comitê de Ética e Pesquisa da Universidade de Vassouras, sob parecer $n^{\circ}$ 3.573.069, de 13 de setembro de 2019 . 
A Coleta de dados foi realizada pela técnica de entrevista em grupo focal, apropriada a pesquisas qualitativas, onde se pretende explorar um "foco", ou seja, um ponto em especial. ${ }^{7}$ Considera-se que possibilita, por meio de um fórum de discussão, a construção de um conhecimento coletivo do grupo.

A amostra foi do tipo intencional, determinada pelo objetivo do estudo num grupo o mais homogêneo possível. O cenário de pesquisa inicialmente foi em um Hospital Geral de ensino do interior do estado do RJ, mas devido a pandemia em função da COVID19 ocorrida neste ano de 2020, o estudo precisou se submeter a uma nova abordagem dos sujeitos e para dar prosseguimento a pesquisa, utilizamos uma ferramenta de videoconferência.

A pesquisa ocorreu em dois momentos distintos, o primeiro encontro foi realizado no início de março de 2020 período antecedente a pandemia em um dos anfiteatros de um hospital geral de ensino e esta reunião contou com a participação de 03 (três) enfermeiros desta referida instituição. O segundo encontro realizou-se por meio da plataforma digital e tivemos a participação de 05 (cinco) enfermeiros.

Os profissionais desta pesquisa trabalham nos setores de Oncologia, Pediatria, Ginecologia e Obstetrícia, Hemodinâmica Clínica Médica, e uma das participantes encontra-se no Núcleo de Segurança do Paciente da Instituição. O tempo em que exercem a profissão no estabelecimento varia de 03 a 20 anos. Buscou-se a composição homogênea selecionando apenas profissionais enfermeiros para o estudo e buscamos preservar as características heterogêneas, num balanço entre uniformidade e diversidade.

\section{Análises e Discussão}

Através da análise da fala dos participantes desta pesquisa foi possível traçar 12 unidades de significação: Protocolos assistenciais como ferramenta de segurançado paciente; as ações de segurança proporcionam ambiente seguro ao profissional; incidente como problema de saúde em qualquer nível de gravidade; importância do registro/notificação na segurança do paciente; núcleo de segurança do paciente: órgão de capacitação e de integração das equipes; lacuna na comunicação; medidas para prevenir a perda de informação dos pacientes; "erros"= aceitabilidade X inaceitabilidade; Política Nacional de Segurança do Paciente: Um novo desafio profissional; interdisciplinaridade na segurança do paciente; metodologias aplicadas na prevenção de erros/ incidentes; e demanda como gerador de incidentes.

A partir do fechamento destas unidades, traçamos a análise da narrativa dos profissionais entrevistados, dispostas em categorias, objetivou-se construir e apresentar as considerações dos profissionais relacionados a temática "Segurança do Paciente" e "Cultura de Responsabilização".

A realização da pesquisa em dois momentos distintos permitiu mensurar a temática na perspectiva dos profissionais em um panorama habitual, e em um panorama de crise.

Para se mensurar o nível de conhecimento que os participantes apresentam sobre a cultura de segurança do paciente e cultura de responsabilização foi discutido com os grupos a Política Nacional de Segurança do Paciente e suas vertentes (como a institucionalização de sua cultura).

A Segurança do Paciente é reconhecida pelos profissionais como uma prática posterior a sua formação e que por isso sua implementação ainda encontra certa dificuldade e resistência, mas percebe-se que a instituição tem empenhado recursos e esforços para sua implementação e que os profissionais, à medida que vão se familiarizando, acabam aderindo de uma melhor maneira.

A segurança do paciente além das 06 metas propostas internacionalmente, visa ações profissionais e institucionais que gerem um ambiente de trabalho estável e seguro não somente aos pacientes, mas também aos profissionais que exercem determinada assistência. Neste eixo notou-se, a partir da fala dos profissionais, que a essência dessas metas, estão no cotidiano de trabalho, no entanto são materializadas em forma de protocolos organizacionais e institucionais, que acabam por limitar seu alcance em proporções maiores, visto que alguns desses protocolos ainda são majoritariamente setoriais.

Ao trazer a temática sobre erros que possam vir a ocorrer na assistência em saúde, ambos os grupos relatam que existem um ambiente favorável a cultura de segurança do paciente e que eles se sentem amparados ao errarem, no entanto um dos grupos ressaltou a ideia de que o erro ainda é visto como algo "inaceitável", que de maneira alguma deve ocorrer, inferindo que apesar da instituição não possuir a cultura punitiva ou de "culpabilização" esta, ainda permeia o imaginário dos Enfermeiros participantes da pesquisa.

Desta forma ao se pensar em segurança do paciente, não há como desvincular sua cultura, que destaca que a punição deve ser substituída pela oportunidade de se aprender com os erros, ou seja, mediante determinada falha na assistência, medidas de prevenção e proteção devem sem traçadas, desde o profissional que está na ponta do cuidado até normas e padrões organizacionais. ${ }^{5}$

Ao discutir sobre Núcleo de Segurança do Paciente, notou-se uma dependência dos participantes por esta instância, percebeu-se que grande parte dos processos realizados nos setores parte de ações e estratégias estabelecidas pelo NSP, e que há uma personificação deste setor nas profissionais que nele 
atuam, onde não foi possível identificar na visão dos participantes o núcleo como órgão que regulamenta e estabelece os processos de trabalho afim de mitigar incidentes, articular os serviços ofertados e promover a qualidade do atendimento seguro ${ }^{8}$, embora o núcleo desta instituição desenvolva ações que visem tais objetivos.

Dentre estas ações, a notificação e capacitação foram as mais citadas pelos participantes desta pesquisa A primeira, é bastante difundida, no entanto não foi possível perceber durante as discussões se há uma análise destas notificações e o que tem sido identificado nos processos de trabalho, para se traçar intervenções necessárias a nível institucional, apesar de ter sido citados situações em que o núcleo age pontualmente.

A segunda, é ofertada a todos os profissionais da instituição, no entanto, segundo a fala de um dos participantes, o comparecimento nestas capacitações irá depender da demanda e complexidade do atendimento prestado em seus setores, e quanto a esses profissionais que não são contemplados, estes possuem suas demandas atendidas pelo Núcleo.

Tratando-se de incidentes, que é um dos focos em segurança do paciente, estes são vistos pelos participantes da pesquisa, como um determinado fato que leva ao dano ao paciente, embora isto se aplique a uma de suas classificações, a literatura ainda os classifica como uma circunstância de potencial risco, que pode ou não causar dano ao paciente, ou seja, não se configura apenas quando ocorre um evento adverso, mas também quando ocorre uma falha na assistência ${ }^{~}$.

Outro ponto importante a ser destacado, é que embora haja uma boa comunicação percebida entre os setores e entre os profissionais e o núcleo, algumas informações se perdem: seja na passagem de plantão ou pela própria demanda dos serviços. É percebido através da fala dos profissionais que o registro de informações embora seja de extrema importância, às vezes tornase deficiente por fatores intrínsecos e extrínsecos aos processos de trabalho.

$\mathrm{Na}$ tentativa de suprir tal deficiência, hoje eles acabam utilizando outros recursos como o uso de aplicativos (WhatsApp). Isso nos leva a reflexão de que a comunicação efetiva é uma das principais barreiras aos erros e uma ação eficaz para a segurança do paciente, mas, ainda precisa ser trabalhada e discutida de maneiras a contemplar essas falhas.

Apesar da pesquisa ter sido realizada com apenas profissionais graduados em Enfermagem, a própria legislação que regulamenta a Política Nacional de Segurança do Paciente, destaca a necessidade de haver o envolvimento de outros profissionais, e isto é ressaltado na fala dos participantes através do destaque da importância da interdisciplinaridade na segurança do paciente, pois ela significa dizer que todos os profissionais são importantes para que a segurança e sua cultura de fato sejam implementadas e exercidas, o foco central retorna ao paciente, onde ele se torna o verdadeiro ator e beneficiário do cuidado.

Por fim, é notório não apenas pela fala dos profissionais, que a Segurança do Paciente nunca foi tão essencial como no momento atual que estamos vivendo. A Pandemia pela COVID-19 exigiu uma reorganização dos processos de saúde pelas equipes atuantes nas instituições públicas e privadas.

Os processos de enfermagem e de trabalho norteados pelos princípios que regem a segurança do paciente e sua cultura são essenciais para que ações seguras sejam traçadas a fim de se garantir a qualidade da assistência, seja em tempos de crises ou não.

\section{Considerações Finais}

A pesquisa foi realizada a fim de reavaliar a cultura de responsabilização em um ambiente hospitalar visando a correlação entre erros e riscos e surgiu por meio do interesse de construir e apresentar as considerações dos profissionais relacionadas a "Segurança do Paciente" e a "Cultura de Responsabilização". O desenvolvimento da pesquisa foi enriquecedor, pois permitiu que traçássemos um novo olhar sobre a cultura de segurança do paciente nos ambientes de saúde em dois momentos distintos.

Por meio do estudo pôde-se perceber que há um conhecimento superficial sobre a temática pelos profissionais entrevistados e que o núcleo é simbolizado na figura das enfermeiras que o gerenciam, embora haja um clima favorável à implementação das ações voltadas à Segurança do Paciente e de sua Cultura.

Conforme a fala trazida pelos profissionais, evidenciou-se a necessidade de que tal unidade seja dotada de uma equipe multiprofissional para que o olhar seja ampliado e haja uma "despersonificação", sendo promovida desta maneira uma maior articulação e integração dos serviços aos profissionais.

As notificações foram apresentadas como estratégia que tiveram grande adesão dos profissionais de Enfermagem e devem continuar sendo estimuladas, mas os resultados dessas notificações precisam ser sentidos pela equipe de saúde de forma mais objetiva, uma vez que não foi possível perceber a clareza dessas informações na fala dos profissionais.

A atual situação que vivemos decorrente da Pandemia do COVID-19, fez com que os profissionais da saúde buscassem tecnologias de comunicação, e como apresentado pelo grupo, agilizou o trabalho e melhorou a comunicação e integração das equipes. Portanto, destaca-se que as tecnologias utilizadas neste momento possam ser aprimoradas para outras atividades a fim de manter a comunicação efetiva, ressaltando que a comunicação falada e registrada não deve ser substituída e sim complementada. 
Nota-se, portanto, que diante da magnitude e complexidade da temática, o estudo possibilitou mensurar o conhecimento dos profissionais acerca da Segurança do Paciente e Cultura de Responsabilização e levar a reflexão do tema pelos enfermeiros e pelas pesquisadoras.

O estudo em Segurança do Paciente não se esgota nesse momento, e sugerimos diante a explanação dos enfermeiros, que o NSP busque a revisão em suas atribuições e atividades de gestão. As propostas indicadas foram pensadas tendo em vista a fala dos profissionais e a realidade da Instituição que atuam, e devem ser entendidas como estratégias que visam a melhoria da prática das ações voltadas a Segurança do Paciente.

\section{Referências}

1. Corrigan JM. Crossing the quality chasm: A new health system for the 21 st century. 2001 .

2. WHO, World Health Organization. World alliance for patient safety: forward programme 2005. Geneva; 2004.

3. Brasil, Ministério da Saúde. Agência Nacional de Vigilância Sanitária. Segurança do paciente e qualidade em serviços de saúde. Boletim Informativo 1. Brasília, DF; 2011.

4. EPSJV, Escola Politécnica de Saúde Joaquim Venâncio [Internet]. Rio de Janeiro: Fiocruz; c2019 [acesso em: 22 ago 2020]. Disponível em: http://www.sites.epsjv.fiocruz.br.

5. Brasil, Ministério da saúde (BR) Portaria $n^{\circ} 529$, de $1^{\circ}$ de abril de 2013. Institui o Programa Nacional de Segurança do Paciente. Diário Oficial União. 2 abr 2013.

6. OMS, Organização Pan-Americana da Saúde (OPAS). Portfólio de cooperação técnica OPAS/ OMS. Brasília; 2018.

7. Victoria C, Knaut DR, HASSEN MN. A pesquisa qualitativa em saúde: Uma introdução ao tema. Porto Alegre: Tomo Editorial; 2000.

8. Brasil, Ministério da Saúde, Agência Nacional de Vigilância Sanitária. Resolução RDC n. ${ }^{\circ}$ 36, de 25 de julho de 2013. Institui ações para a segurança do paciente em serviços de saúde e dá outras providências.

9. WHO, World Health Organization. Estrutura conceitual da Classificação Internacional sobre Segurança do Doente. Relatório Técnico Final. Direção Geral da Saúde. Geneva; 2011. 